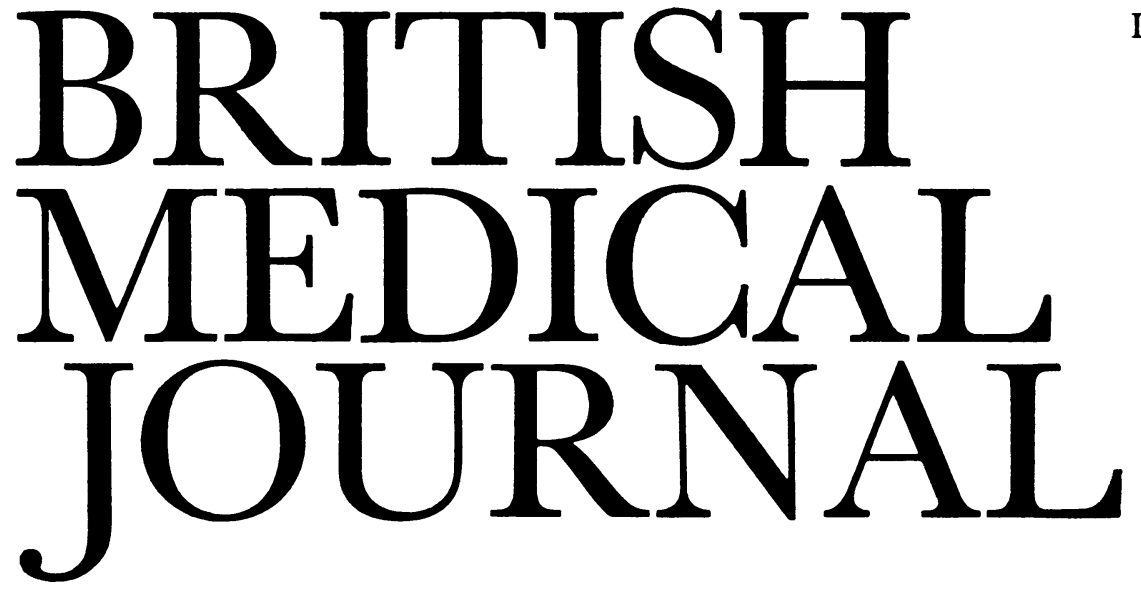

LONDON, SATURDAY 11 AUGUST 1984

\title{
Snoring and its treatment
}

"Snore events" are defined in a recent study as inspiratory, raspy, snortlike sounds, and nine habitual snorers each emitted 500 to 1300 such noises nightly. ${ }^{\prime}$ No one who has slept beside a snorer needs any definition for their recognition, or any convincing that a remedy would be welcome. And one is on offer: continuous positive airway pressure applied through the nose-nasal CPAP.

Snoring occurs when an excessive inspiratory effort is overcoming obstruction of the upper airway. The walls of the oropharynx tend to collapse with the generalised muscular relaxation which is particularly profound in rapid eve novement sleep. Altered drives to breathing result in a s. Iodicity comparable to the Cheyne-Stokes pattern; in the waning (or even apnoeic) phase the fall in blood oxygen and the rise in carbon dioxide can be considerable before they stimulate respiration; the diaphragm then "wakes up" in advance of the upper airway muscles, so that the inspiratory effort may simply suck the limp walls of the oropharynx together. Progressively stronger efforts succeed in opening the airway and pulling in air, setting up the vibrations of a snore. If the upper airway is further obstructed by a blocked nose or by enlarged adenoids the collapse of the oropharynx on inspiratory effort is exaggerated. ${ }^{2}$

This description-and most reports of the use of nasal CPAP_relate to the obstructive sleep apnoea syndrome ${ }^{3}$; but where benign snoring ends and the syndrome begins is arbitrary. Excessive snoring is always a feature of the syndrome but its diagnosis also requires at least 30 episodes a night of obstructive apnoea (respiratory effort without airflow) each lasting at least 10 seconds. These are related to troughs of arterial oxygen saturation. Patients with the obstructive sleep apnoea syndrome also have symptoms during the day-notably somnolence-attributable to their loss of normal sleep resulting from repeated arousal.

In one recent study four of the nine people recruited for treatment simply as severe snorers ${ }^{1}$ were found to qualify as mild cases of the obstructive sleep apnoea syndrome; another one who had no symptoms other than snoring had a full quota of apnoeas. ' Clearly the boundaries are indistinct. Even two simple snorers showed minimum oxygen saturations of $85 \%$, which implies $\mathrm{PaO}_{2}$ of under $60 \mathrm{~mm} \mathrm{Hg}$ (8

\footnotetext{
(C) BRITISH MEDICAL JOURNAL 1984. All reproduction rights reserved.
}

$\mathrm{kPa}$ ). An Edinburgh sleep study which included a control group of 20 ostensibly healthy people showed two who had more than the specified occurrences of obstructive apnoea and desaturation needed to qualify for the obstructive sleep apnoea syndrome. ${ }^{4}$

So heavy snorers may need to be treated not only because they are a nuisance but also because they may have, or may develop, the full obstructive sleep apnoea syndrome with its hazards of nocturnal hypoxaemia, systemic and pulmonary hypertension, and a variety of daytime disorders.

Whether a patient has the obstructive sleep apnoea syndrome or is simply a snore $i$, the rationale of treatment is first to clear any obvious obstruction; snoring children in particular may warrant surgical treatment despite lack of other symptoms. ${ }^{5}$ The large body of mostly male middle aged snorers are more likely to have a functional disorder of the oropharynx. At worst, it can be bypassed: for the full blown obstructive sleep apnoea syndrome, until now the only reliably successful treatment has been tracheostomy. ${ }^{6}$ It would be a harsh wife and a brave surgeon who would seek and concede tracheostomy for an otherwise asymptomatic snorer, though it would surely cure him. Less drastically the contribution of the weight of the jaw and the relaxing genioglossus-the falling back tongue-may be modified by preventing the snorer from lying on his back. (Surprisingly, there are very few published reports on the effect of posture; one does state that it made no difference to the incidence of obstructive episodes in five patients with the obstructive sleep apnoea syndrome. ${ }^{7}$ )

The rational alternative is to attempt to overcome the collapsing tendency of the airway by expanding it with raised air pressure. This can be done by fitting a personalised well sealed mask to the nose, delivering air to it from a compressor, and maintaining a pressure of $5-10 \mathrm{~cm} \mathrm{HO}$ throughout the breathing cycle by adjusting the resistance at the outlet. This nasal CPAP has cured the obstructive sleep apnoea syndrome dramatically in the hands of those who have used it most: further to a report in 1981 of complete relief in all of five patients,' Sullivan's group recently claimed 50 successes with half the patients continuing treatment at home. ${ }^{8}$ Other groups have also found 
the method appropriate; another 10 successes were reported but with a warning - in one instance CPAP stopped the obstructive episodes and restored breathing which was regular but so inadequate that hypoxaemia became dangerously worse. ${ }^{9}$ The patient was exceptional in having chronic respiratory disease, and such patients are quite different from those with the obstructive sleep apnoea syndrome. ${ }^{4}$ Nevertheless this one report suggests that no patient should be put on a CPAP regimen unless his response to it has been monitored with continuous measurement of oxygen saturation.

Another group using CPAP for obstructive sleep apnoea syndrome has now confirmed by computed tomography the assumption that the benefit is due to widening of the upper airway and claimed $100 \%$ success in 16 patients. ${ }^{10}$ Others, however, have found a similar improvement in patients with the obstructive sleep apnoea syndrome when they used only expiratory positive airway pressure, arguing that the improvement with either method might be by way of increasing functional residual capacity. ${ }^{.1}$

There seems no danger attached to nasal CPAP (with the exception quoted). ${ }^{9}$ Patients mostly tolerate it well and can sleep with it; it is not excessively complicated or expensive; and, indeed, there are good reasons to consider its use for recalcitrant snorers. Though they may not complain of daytime symptoms the enhanced quality of their sleep might improve their well being-as well as the peace and quiet of the home. With this treatment Berry and Block reduced the mean number of snores in their patients from 1000 plus to 23 a night, ${ }^{1}$ with similar dramatic falls in the number of apnoeic or hypopnoeic events and the number and severity of desaturations.

It should not be too difficult to arrange a snore recorder for microcomputer analysis, so that the number of snores a night could be counted: the number appears to be some guide to the incidence of apnoea or hypopnoea and thus to the risk of the obstructive sleep apnoea syndrome and the need for treatment.

Until there are more centres in Britain with an interest in sleep disorders and equipped for "polysomnography," it is unfortunately difficult to know just who might be eager to apply themselves to the problem of providing nasal CPAP. It could well improve the quality of life for those who in the night, like Dionysius, make "much adoe to take a breath." 12

\section{SHEILA JENNETT}

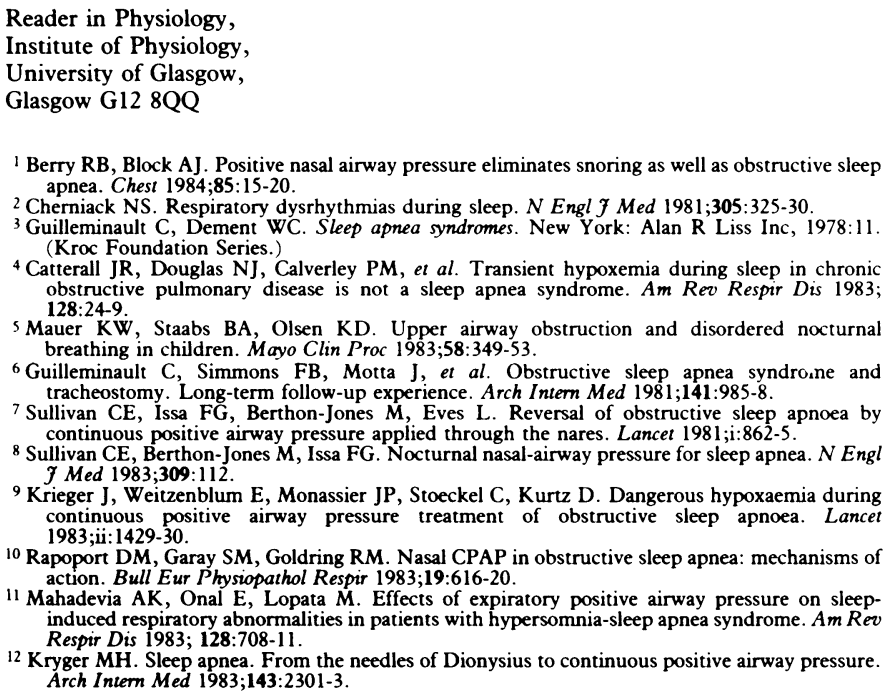
12 Kryger MH. Sleep apnea. From the needles of Dionysius to continuous positive airway pressure.
Arch Intern Med 1983;143:2301-3.

\section{A fair trial?}

Randomised clinical trials have become accepted as the standard procedure for comparing treatments, particularly for evaluating a new treatment against either an existing treatment or placebo. The many publications on this subject are continuing to grow in numbers but some aspects of trials are still not handled well. One important example is the assessment of the comparability of the patients in different treatment groups. Until recently this has received surprisingly little attention, ${ }^{1}$ but an important paper by Lavori et al has considered the problem in some depth. ${ }^{2}$

In a clinical trial, randomisation ensures that the allocation of treatments to patients is left purely to chance. There is, however, no guarantee that the patients in the different treatment groups will be similar with respect to characteristics such as age, severity of disease, history, and so on. Despite randomisation, quite probably patients with a particular characteristic will not be equally common within each group. Imbalance of such baseline variables is clearly undesirable, as the essence of a controlled trial is the comparison of groups differing only as regards their treatment. Such imbalance really matters, however, only for variables with prognostic importance.

The usual and eminently sensible procedure is to begin the analysis of a trial by comparing the treatment groups with respect to several baseline variables. The fact that this is so common implies that most research workers realise that randomisation does not guarantee that the groups will be comparable. Unfortunately, the next step is often to perform significance tests, comparing these baseline characteristics in the different groups. Although carrying out such tests is not wrong, they are nearly always misinterpreted, perhaps seriously.

Presumably the intention behind performing significance tests to compare baseline variables is to see whether an observed difference is important. What such tests actually assess, however, is the probability that the observed difference (or a greater one) could have occurred by chance when in reality there was no difference. A moment's thought will show that when comparing baseline characteristics we know that the null hypothesis is true-because the treatment groups were produced by randomisation. If the randomisation was performed properly a difference of any sort between the groups before treatment must necessarily be due to chance. Tests of significance are thus testing only the fairness of the randomisation. We would expect one test in 20 to give a significant result with $\mathrm{p}<0.05$, and this is true however many patients are studied. We should not conclude from the lack of statistical significance that imbalance in a baseline variable did not affect the outcome of the trial; the comparability of treatment groups cannot be assessed in this way.

In very large trials this common error of interpretation is unlikely to be serious, but in smaller trials some quite substantial differences may occur by chance. Unless these differences are shown to be statistically significant they are nearly always ignored-as a result of the faulty reasoning described. ${ }^{2}$ An extreme example of this was in a clinical trial of patients with acute myocardial infarction where the prevalence of previous infarction in the two groups was $7 \%$ and $30 \% .^{3}$ Even when differences are found to be statistically significant their possible importance is still often ignored. ${ }^{45}$ Armitage has suggested that major baseline differences will be rare. ${ }^{6}$ This is certainly true for large trials, but far too many clinical trials are performed on very small numbers of 